\title{
Retrograde Intrarenal Surgery, Another Minimally Invasive Procedure for Upper Tract Urolithiasis: Our initial Experience at a Tertiary Care Hospital
}

Syed Sajjad Nazir ${ }^{1 *}$, Shabir Ahmad Mir $^{2}$, Shahnawaz Rasool ${ }^{3}$, Fuad Sadiq Baqal ${ }^{2}$, Tanveer Iqbal Dar ${ }^{4}$, Omar Saleem Akhter $^{5}$, Javeed Ahmad Magrey ${ }^{6}$

${ }^{1}$ Associate Professor \& Head, Department of Urology, Super Speciality Hospital, Government Medical College Srinagar, Jammu and Kashmir, India

${ }^{2}$ DNB Resident, Department of Urology, Super Speciality Hospital, Government Medical College, Srinagar, India

${ }^{3}$ Senior Resident, Department of Urology, Super Speciality Hospital, Government Medical College, Srinagar, India

${ }^{4}$ Assistant Professor, Department of Urology, Super Speciality Hospital, Government Medical College, Srinagar, India

${ }^{5}$ Lecturer, Department of Urology, Super Speciality Hospital, Government Medical College, Srinagar, India

${ }^{6}$ Consultant, Department of Urology, Super Speciality Hospital, Government Medical College, Srinagar, India

DOI: $10.36347 /$ sjams.2020.v08i05.021

| Received: 05.05.2020 | Accepted: 12.05.2020 | Published: 14.05.2020

*Corresponding author: Syed Sajjad Nazir

Abstract

Original Research Article

Background: As the advancement in technology and miniaturization of equipments, RIRS provides an alternative way to PCNL by minimizing the risks related to PCNL in the treatment of Urolithiasis. Objective: The aim of this study was to explore our initial experience with RIRS in terms of feasibility, the efficacy and safety for the management of Urolithiasis. Materials and Methods: This prospective study was carried out in the Department of urology Super Specialty Hospital Shereenbagh, Government Medical College Srinagar over a period of 6 months from October 2019 to April 2020. This study comprised of patients admitted with upper tract stones who opted for RIRS after being properly explained the procedure. Follow up NCCT KUB was obtained after 5 weeks of RIRS to look for the clearance of stone. Results: A total of 46 patients were enrolled. The age of the Patients ranged from 26 to 63 years with a mean age of 44.23 years. Out of total 46 patients, preoperative DJ stenting was ensured in 42 patients. In two patients RIRS was converted to PCNL. In our study stone free rate was $81.8 \%(36 / 44)$ after the single session of RIRS. Residual stone was higher in cases of lower calyceal stones, stones in multiple calyces and stone burden $>2 \mathrm{~cm}$. Residual stones 3-4 $\mathrm{mm}$ were present in 14 patients and $>5 \mathrm{~mm}$ in 2 pts.. Operative time ranged from 55 minutes to 195 minutes (average time 100.57 minutes). Patients were discharged after $36 \mathrm{hrs}$. There were no major complications in our study. Conclusion: RIRS has not only the advantage of being minimally invasive, but also is associated with low rate of complications, less morbidity and good stone free rate and short hospital stay. RIRS appears to be a safe and effective alternative to PCNL for upper tract stones.

Keywords: RIRS, Laser, SFR, UAS, PCNL.

Copyright @ 2020: This is an open-access article distributed under the terms of the Creative Commons Attribution license which permits unrestricted use, distribution, and reproduction in any medium for non-commercial use (NonCommercial, or CC-BY-NC) provided the original author and source are credited.

\section{INTRODUCTION}

With the development of flexible Ureteroscope and technical advancements, Retrograde intrarenal surgery (RIRS) performed using a flexible ureterorenoscope marked the beginning of a new era in urology. RIRS renders smaller kidney stones more accessible and upper urinary tract tumors treatable, using minimally invasive methods [1]. RIRS was first used to treat small kidney stones [2].The approach attracted a great deal of attention and it was suggested that larger stones could also be treated, albeit over longer operative times. Initially, medium and then larger stones were treated via RIRS [3]. Percutaneous nephrolithotripsy (PCNL) is a gold standard procedure for large kidney stones with a potential morbidity of bleeding, which might need angioembolization, and also has certain limitations in patients with bleeding diathesis [4], obesity and malrotated kidneys. Retrograde intrarenal surgery (RIRS) is a less morbid procedure than PCNL [5], though the Kidney stones greater than $2 \mathrm{~cm}$ have long been treated with percutaneous nephrolithotomy (PNL) $[6,7]$.

Recent studies reported stone-free rates from $77 \%$ to $>90 \%$ for RIRS of renal stones and $62 \%$ to $85 \%$ for the management of lower pole stones [7-11]. Furthermore, several studies have reported significant success rates with RIRS in the management of large renal stones [12]. Recently, studies reporting the 
efficacy of RIRS in lower pole stones have increased [13]. Under this background we conducted this study to look for the feasibility, efficacy and safety of RIRS for the management of Urolithiasis in our set-up.

\section{MeTHODS}

This prospective study was carried out in the Department of urology Super Specialty Hospital Shereenbagh, Government Medical College Srinagar over a period of 6 months from October 2019 to April 2020. This study comprised of patients admitted with the diagnosis of nephrolithiasis and upper ureteric stones who opted for RIRS after being properly explained the procedure.

\section{Aim}

The aim of this study was to explore our initial experience with RIRS in terms of feasibility, the efficacy and safety for the management of upper tract calculi.

\section{Inclusion Criteria}

- Patients with upper tract calculi of age $>15$ years and above

- Patients with renal and/or upper ureteric stones (single/multiple)

- Stone burden $<1$ to $3 \mathrm{~cm}$

- $\quad$ Patients with stones in any of the calyces; $>1$ calyces; pelvis; and upper ureteric stones

- Previous history of URS or ESWL or Open surgery.

- Patients with comorbidities such as diabetes, hypertension, and patients on anticoagulants.

- Anatomically abnormal kidneys such as solitary kidney, horseshoe kidney, and ectopic kidney.

\section{Exclusion Criteria}

- Patient preference

- Age group $<15$ years

- Stone burden more than $3 \mathrm{~cm}$

- Complete staghorn calculi.

All patients were subjected to the routine preoperative workup, negative urine culture and plain CT KUB with contrast enhancement and, data was collected. A total of 46 patients were enrolled during the period of our study. Among these preoperative DJ stenting was ensured in 42 patients $3-5$ days prior to procedure. All patients were subjected to general anesthesia. Cystoscopy followed by ureteroscopy with a Semi-rigid 7F ureteroscope was performed at the start of procedure. This initial ureteroscopy helps to dilate the ureter, manage any stone fragment, to discover any undiagnosed pathology like malignancy and ureteral condition. UAS (ureteral access sheath) 10/12 F is placed over a 0.038 teflon guide wire under $\mathrm{C}$ arm. Irrigation tubing is attached to the flexible digital ureterorenoscope from Olympus to provide the clear vision using a guarded pressure pump. All calyces are being inspected. Basket is used for stones or fragments $<1 \mathrm{~cm}$ for repositioning to appropriate location. A 230 micron laser fiber is used with the Holmium YAG laser of 35 watts of Karl Storz make (Calculase III). The Laser settings used were as follows: Fragmentation $\rightarrow$ high energy (1-2 J) with low frequency (3-5 Hz)with short pulse duration; Dusting $\rightarrow$ Low energy (0.2-0.5 J)with High frequency ( $10-20 \mathrm{~Hz}$ ) with long pulse duration and for Popcorn effect $\rightarrow$ High energy ( $1 \mathrm{~J}$ ) with High frequency $(10-20 \mathrm{~Hz})$ with long pulse duration. A few stone fragments are removed by basket for analysis. Finally contrast study is performed for any intravasation and pelvicalyceal system is reexamined with flexible ureterorenescope. We placed DJ stent at the end in all patients. At the end of the procedure access sheath was removed under flexible ureterorenoscope direct vision to see any ureteric injury due to insertion of ureteral access sheath. Patients are being monitored postoperatively for any complication. We usually discharged our patients after 36 hrs. Follow up NCCT KUB was obtained after 5 weeks of RIRS to look for the clearance of stone burden. Stone size $\geq 3 \mathrm{~mm}$ on follow up NCCT KUB was considered as residual stone, while a size of $>5 \mathrm{~mm}$ was taken as significant.

\section{Statistical Methods}

The recorded data was compiled and entered in a spreadsheet (Microsoft Excel) and then exported to data editor of SPSS Version 20.0 (SPSS Inc., Chicago, Illinois, USA). Continuous variables were expressed as Mean \pm SD and categorical variables were summarized as frequencies and percentages.

\section{RESULTS}

A total of 46 patients were enrolled during the period of our study. The age of the Patients ranged from 26 to 63 years with a mean age of 44.23 years. Out of these 27 were males and 19 were females. Twenty-eight patients had right sided procedures and 18 patients had left sided procedures. Out of total 46 patients, preoperative DJ stenting was ensured in 42 patients. Intraoperative views of RIRS are depicted in Figures 1 to 2, and stone dust passing through perurethral Foley's catheter after RIRS is shown in Figure-3. Figure-4 shows RGP demonstrating complete duplication with infundibular stenosis and calyceal calculi and figure 5 depicted fluoroscopic view during RIRS.

In two patients RIRS was converted to PCNL. Three patients had stones with infundibular stenosis, one in upper calyx, one in lower calyx, and another had patient had complete duplication of pelvicalyceal system with stone present in the upper blind calyx of superior moeity. Patient with upper calyceal stone with infundibular stone was managed by RIRS after reestablishing the communication with help of the Laser fibre. The patient with complete duplication (Figure-4) of pelvicalceal system having stone in the superior 
moiety in upper calyx (stenosed neck) was dealt with RIRS as in the above case.

One patient of infundibular stenosis with a stone obstructing the calyceal neck in the lower pole calyx leadindg to cystic dilatatation with stones in the cystic cavity was converted to PCNL because of technical difficulty. One more patient with lower calyceal stone was converted to PCNL because of difficult anatomy due to previous open renal stone surgery. One patient with stone in the pelvis had angiomyolipoma in the lower pole and was successfully managed with the RIRS without any complication. Stone free rate (SFR) and time taken is analysed in Table 1 to 3 .

Overall average operative time $=100.57$ minutes. Average operative time in the initial half of our patient cohort was 113 minutes whereas the average operative time in the latter half of our patient cohort was 88.14 minutes. Hematuria cleared up in majority of the patients within 6-8 hours of surgery and in all patients within 14 hours. Pain score on the day of surgery and the first POD was calculated using the VAS score and ranged from 2-7 with an average 3.06. Analgesic required was 2 doses of paracetamol $1 \mathrm{~g}$ i.v on the day of surgery. Three patients complained of the pain in the flank region persistently for 3-4 days and were managed with oral analgesics. One patient developed steinstrass postoperatively after stent removal and developed fever on and off for 10 days and was managed initially with antibiotics. Steinstass did not resolve until 5 weaks postoperatively and was managed then by second setting of RIRS. Residual stones 3-4 $\mathrm{mm}$ were present in 14 patients and were managed by observation policy while two cases with residual stone $>5 \mathrm{~mm}$ including the patient with steinstrasse were managed by second RIRS after 5 weeks of index RIRS. We discharged 44 patients after $36 \mathrm{hrs}$. Patients were advised to resume their routine work after 4-5 days of surgery in most of the cases. Stent were removed after 2 weeks of surgery.

Table-1: Stone-free rate according to stone location after index RIRS procedure

\begin{tabular}{|l|l|l|l|}
\hline Location of stones & Number of patients & $\begin{array}{l}\text { No. of patients with } \\
\text { residual stones }\end{array}$ & Stone free rate (\%) \\
\hline Upper calyx & 3 & 0 & 100 \\
\hline Middle calyx & 3 & 0 & 100 \\
\hline Lower calyx & 10 & 4 & 60 \\
\hline Pelvis & 4 & 0 & 100 \\
\hline Multiple calyces & 15 & 4 & 73.33 \\
\hline Upper ureter & 9 & 0 & 100 \\
\hline Total & 44 & 8 & 81.8 \\
\hline
\end{tabular}

Table-2: Stone-free rate according to stone burden after index RIRS procedure

\begin{tabular}{|l|l|l|l|}
\hline Stone Burden(cm) & Number of patients & Patients with residual stones & Stone free rate \\
\hline$<1$ & 8 & 1 & 87.5 \\
\hline $1-2$ & 29 & 5 & 82.7 \\
\hline$>2$ & 7 & 2 & 71.4 \\
\hline Total & 44 & 8 & 81.8 \\
\hline
\end{tabular}

Table-3: Operative time ${ }^{*}$ versus stone burden

\begin{tabular}{|l|l|l|}
\hline Stone Burden(cm) & Operative Time(min) & Average time(min) \\
\hline$<1$ & $55-72$ & 64 \\
\hline $1-2$ & $63-124$ & 98 \\
\hline$>2$ & $115-195$ & 153 \\
\hline$*$ operative time=Time taken by the surgeon, i.e., the time was calculated from starting the endoscopic \\
procedure till catheterization. Anesthesia, positioning and preparation time were not included.
\end{tabular}




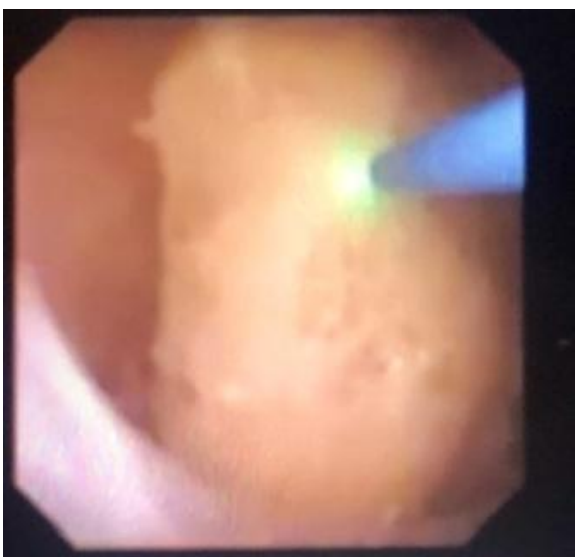

Fig-1: Laser fibre ready to dust the stone

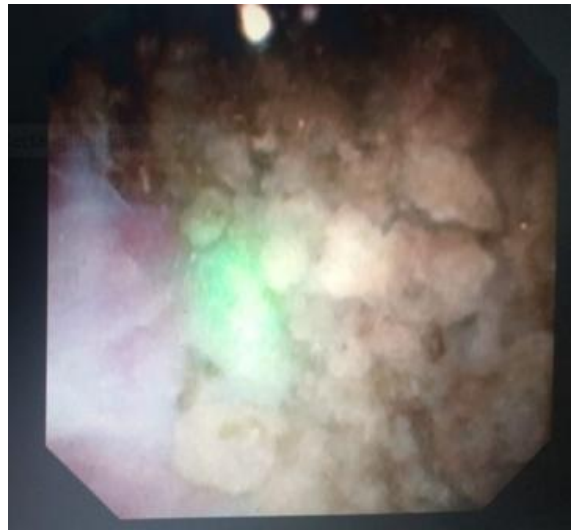

Fig-2: Laser fibre fragmenting the stone

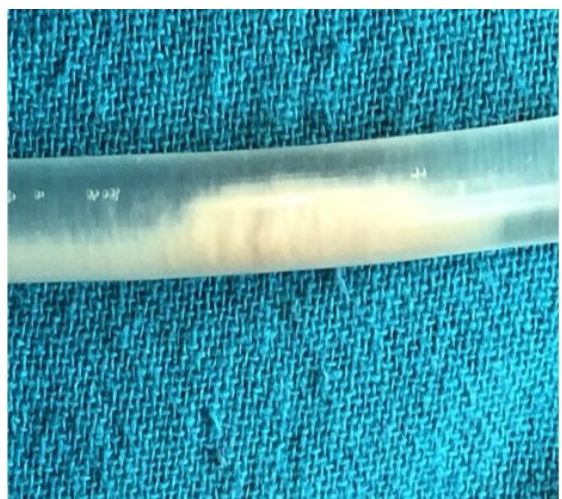

Fig-3: Stone dust passing through perurethral Foleys catheter after RIRS

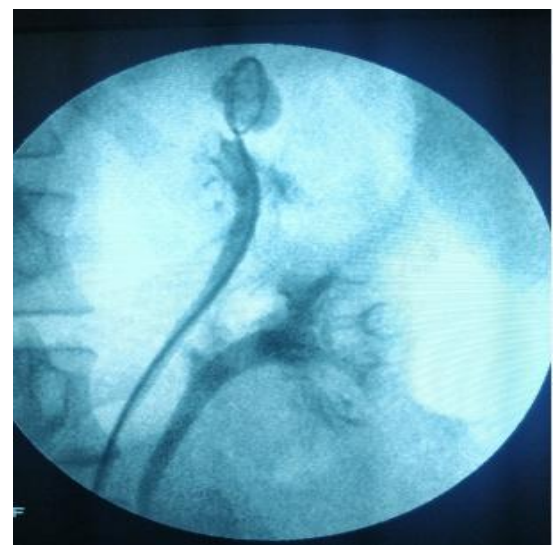

Fig-4: Complete duplication of pelvicalceal RIRS Procedure

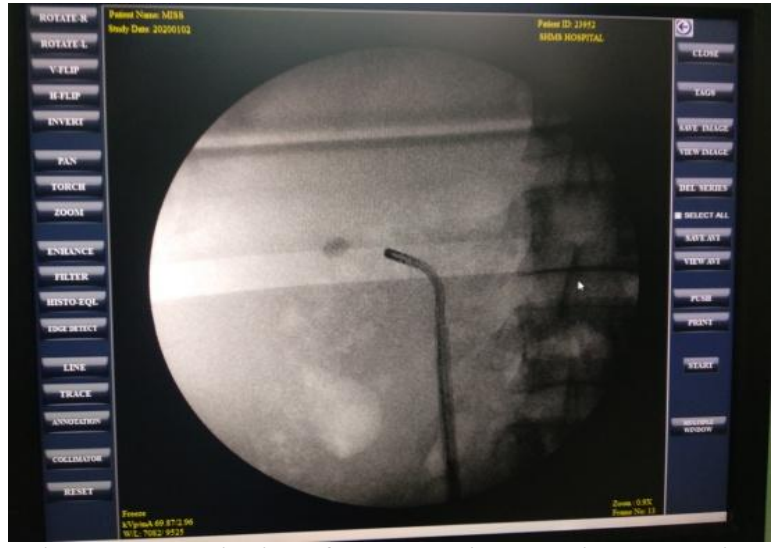

Fig-5: Floroscopic view of system having stone in the superior moiety

\section{DiSCUSSION}

RIRS provides safe and effective method alternative to PCNL in the surgical management of renal or upper ureteric stones, though there remains a debate for stone clearance in case of RIRS as compared to PCNL. There seems to be lesser learning curve for RIRS as compared to the PCNL though cost is an issue in case of RIRS. RIRS is less invasive than PCNL. Grasso and associates [10] have shown the use of RIRS for large renal stones in patients who had comorbid conditions and were not fit for PCNL. Hyams et al., [14] reported using RIRS for the treatment of renal stones with diameters of $20-30 \mathrm{~mm}$ in 120 patients. They achieved $63 \%$ SFR when a stone-free state was defined as no residual stones or only clinically insignificant stone fragments of $<2 \mathrm{~mm}$ were present. If the stone-free state was defined as no residual stone fragments of $<4 \mathrm{~mm}$, the SFR would increase to $83 \%$. Prabhakar et al., [15] reported that RIRS could achieve a $100 \%$ SFR in treating renal stones with an average diameter of $25 \mathrm{~mm}$ after a single or staged procedure. They considered complete clearance, if there were no fragments on USG screening after 3 weeks. Twenty six $(86.6 \%)$ their patients out of 30 had complete clearance in the first sitting and 4 (13.3\%) patients needed re-look flexible ureteroscopy. All four patients had residual fragments less that $6 \mathrm{~mm}$ which needed only basketing; there was no need for fragmentation.

The age of our patients ranged from 26 to 63 years with a mean age of 44.23 years. In our study stone free rate was $81.8 \%(36 / 44)$ after the single session of RIRS. Rate of residual stone was higher in cases of lower calyceal stones, stones in multiple calyces and stone burden $>2 \mathrm{~cm}$. Residual stones $3-4 \mathrm{~mm}$ were present in 14 patients and were managed by observation policy. The two patients with significant residual stone size $(>5 \mathrm{~mm})$ were subjected to second stage of RIRS after 5 weeks of index RIRS with complete clearance. Anatomical factors that affect the failure to access lower pole in fURS were evaluated [16]. Although acute IPA $<30^{\circ}$ and length of infundibulum $>3 \mathrm{~cm}$ were found to be associated with lower SFRs, while width of infundibulum had no effect. Increase in deflection with 
technological developments and improvements in surgical technique have led flexible ureteroscopes to reach lower pole more easily. Repositioning lower pole stones with tipless nitinol baskets to other calyxes that are accessed easily has increased the treatment success of fURS in the management of lower pole stones [17].

In the study by Prabhakar et al., [15], operating time was 45 minutes to 190 minutes (average time 92 minutes) the time was calculated from starting the endoscopic procedure till catheterization. In our study operative time ranged from 55 minutes to 195 minutes (average time 100.57 minutes). Average operative time in initial half of our patient cohort was 113 minutes whereas the average operative time in the latter half of our patient cohort was 88.14 minutes. This is in consistence with short learning curve of RIRS.

Endoscopic stone treatment is more difficult in patients with a history of open surgery due to intra renal anatomic distortion. Osman et al., [18] reported SFRs of $79.2 \%$ and $92.4 \%$ after first and second procedures in 53 patients with an average stone diameter of $14.3 \mathrm{~mm}$. They noted $2(3.7 \%)$ intraoperative complications, including a ureteral perforation and extravasation, and a hemorrhage not requiring blood transfusion; while 9 postoperative complications $(18 \%)$ were noted. In our study, one patient with lower calyceal stone was converted to PCNL because of difficult anatomy due to previous open renal stone surgery causing closure of infundibilium.

With regard to RIRS in infundibular stenosis and stones in calyx diverticulum, Koopman et al., [19] dilated the calyx neck with either balloon dilatator or laser incision, and succeeded to reach the stone in calyx diverticulum in $94 \%$ of 108 patients. General SFR was $90 \%$, while they reported a SFR of $75 \%$ for $2-3 \mathrm{~cm}$ stones with addition of SWL. Chen et al., [20] opened the calyx neck with only laser incision in 43 patients, and had success in 35 patients $(81.4 \%)$ after the first session. Five of the remaining 8 patients were stone-free after the second session, and they reported an overall SFR of $93 \%$ without any major complication. In our study, three patients had stones with infundibular stenosis, one in upper calyx, one in lower calyx, and another had patient had complete duplication of pelvicalyceal system with stone present in the upper blind calyx of superior moeity. Patient with upper calyceal stone with infundibular stone was managed by RIRS after reestablishing the communication with help of the Laser fibre incision. The patient with complete duplication of pelvicalyceal system having stone in the superior moiety in upper calyx (blind) was dealt with RIRS as in the above case. One patient of infundibular stenosis with a stone obstructing the calyceal neck in the lower pole calyx leadindg to cystic dilatation with stones in the cystic cavity was converted to PCNL because of technical difficulty.
In the study by Prabhakar et al., [15], no major complications were reported. All the patients were discharged in $24 \mathrm{hrs} .96 .6 \%$ (29/30) of patients resumed normal duties on the third postoperative day. We discharged 44 patients after 36 hours of the surgery. Patients were advised to resume their routine work after 4-5 days of surgery in most of the cases. There were no major complications in our study except one patient developed steinstrasse after DJ Stent removal who was managed successfully initially with antibiotics and latter on with ReRIRS. Hematuria cleared up in majority of the patients within 6-8 hours of surgery and in all patients within 14 hours. Pain score on the day of surgery and the first POD was calculated using the VAS score and ranged from 2-7 with an average 3.06. One patient in our study with stone in the renal pelvis had angiomyolipoma in the lower pole and was successfully managed with the RIRS without any complication which was unfit for PCNL.

\section{CONCLUSION}

RIRS has not only the advantage of being minimally invasive, but also is associated with low rate of complications, less morbidity and good stone free rate and short hospital stay. Large stone burden and lower calyceal stone location are the important factors deciding low SFR in RIRS. There is minimal requirement of postoperative analgesics. RIRS is better modality than PCNL were angioembolization facilities are not available and also in patients with bleeding diathesis and obesity. Hence, RIRS appears to be a safe and effective alternative to PCNL for upper tract stones up to a stone burden of $3 \mathrm{cms}$, and seems to be potentially safer in certain situations including bleeding diathesis.

\section{Funding: None}

\section{Conflict of Interest: None}

Ethical Approval: not required

\section{REFRENCES}

1. Abdel-Razzak OM, Bagley DH. Clinical experience with flexible ureteropyeloscopy. The Journal of urology. 1992 Dec 1;148(6):1788-92.

2. Bagley DH. Expanding role of ureteroscopy and laser lithotripsy for treatment of proximal ureteral and intrarenal calculi. Current opinion in urology. 2002 Jul 1;12(4):277-80.

3. Breda A, Ogunyemi O, Leppert JT, Lam JS, Schulam PG. Flexible ureteroscopy and laser lithotripsy for single intrarenal stones $2 \mathrm{~cm}$ or greater-is this the new frontier?. The Journal of urology. 2008 Mar 1;179(3):981-4.

4. Watterson JD, Girvan AR, Cook AJ, Beiko DT, Nott L, Auge BK, Preminger GM, Denstedt JD. Safety and efficacy of holmium: YAG laser 
lithotripsy in patients with bleeding diatheses. The Journal of urology. 2002 Aug 1;168(2):442-5.

5. Egilmez $T$, Goren MR. Predicting surgical outcome of percutaneous nephrolithotomy: validation of the Guy's stone score and nephrolithometric nomogram in terms of success and complications. Journal of Clinical and Analytical Medicine, 2015;6(3), 281-286.

6. Segura JW, Patterson DE, LeRoy AJ, Williams HJ, Barrett DM, Benson RC, May GR, Bender CE. Percutaneous removal of kidney stones: review of 1,000 cases. The Journal of urology. 1985 Dec;134(6):1077-81.

7. Michel MS, Trojan L, Rasweiler JJ. Complications in percutaneous nephrolithotomy. Eur Urol, 2007; 51: 899-906.

8. Unsal A, Resorlu B, Atmaca AF, Diri A, Goktug HN, Can CE, Gok B, Tuygun C, Germiyonoglu C. Prediction of morbidity and mortality after percutaneous nephrolithotomy by using the Charlson Comorbidity Index. Urology. 2012 Jan 1;79(1):55-60.

9. Deem S, Defade B, Modak A, Emmett M, Martinez F, Davalos J. Percutaneous nephrolithotomy versus extracorporeal shock wave lithotripsy for moderate sized kidney stones. Urology. 2011; 78: 439-43.

10. Grasso M, Conlin M, Bagley D. Retrograde ureteropyeloscopic treatment of $2 \mathrm{~cm}$ or greater upper urinary tract and minor staghorn calculi. J Urol, 1998; 160: 346-51.

11. Grasso M, Ficazzola M. Retrograde ureteropyeloscopy for lower pole caliceal calculi. J Urol, 1999; 162: 1904-8.

12. Gupta M, Oct Mc, Shah JB. Percutaneous managemen of the upper urinary tract. Campbell-
Walsh Urology, 9th ed. Philaselphia, PA: Saunders Elsevier, 2007; 1544-8.

13. Lingeman JE, Siegel YI, Steele B, Nyhuis AW, Woods JR. Management of lower pole nephrolithiasis: a critical analysis. J Urol, 1994; 151: 663-7.

14. Hyams ES, Munver R, Bird VG, Uberoi J, Shah O. Flexible ureterorenoscopy and holmium laser lithotripsy for the management of renal stone burdens that measure 2 to $3 \mathrm{~cm}$ : a multiinstitutional experience. Journal of endourology. 2010 Oct 1;24(10):1583-8.

15. Prabhakar M. Retrograde ureteroscopic intrarenal surgery for large $(1.6-3.5 \mathrm{~cm})$ upper ureteric renal calculus. Indian J Urol. 2010; 26: 46-49.

16. Jessen JP, Honeck P, Knoll T, Wendt-Nordahl G. Flexible ureterorenoscopy for lower pole stones: influence of the collecting system's anatomy. Journal Endourol, 2014; 28:146-51.

17. Kourambas J, Delvecchio F, Munver R, Preminger GM. Nitinol stone retrieval-assisted ureteroscopic management of lower pole renal calculi. Journal Urol, 2012; 4295: 935-9.

18. Alkan E, Saribacak A, Ozkanli AO, Başar MM, Acar O, Balbay MD. Retrograde intrarenal surgery in patients who previously underwent open renal stone surgery. Minim Invasive Surg, 2015; 2015:198765.

19. Koopman SG, Fuchs G. Management of stones associated with intrarenal stenosis: infundibular stenosis and caliceal diverticulum. Journal Endourol, 2013; 27:1546-50.

20. Chen X, Li D, Dai Y, Bai Y, Luo Q, Zhao Z, Chen $\mathrm{H}$, Zhang $\mathrm{X}$. Retrograde intrarenal surgery in the management of symptomatic calyceal diverticular stones: a single center experience. Urolithiasis. 2015 Nov 1;43(6):557-62. 